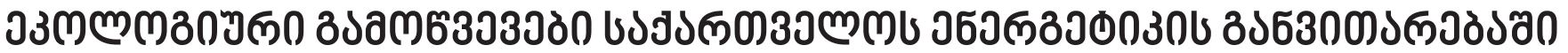

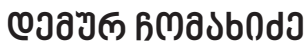

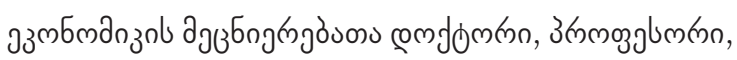

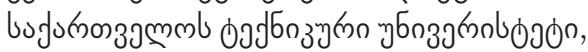

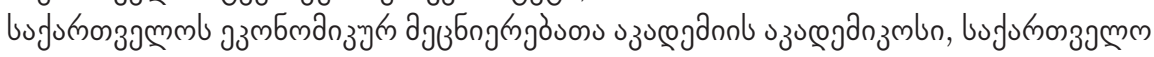

d.chomakhidze@gnerc.org

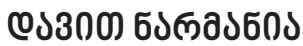

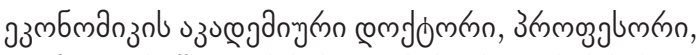

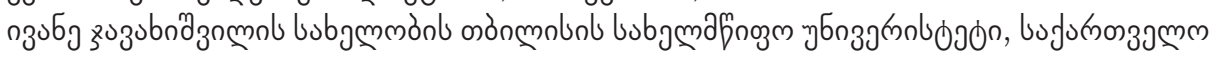

davit.narmania@tsu.ge

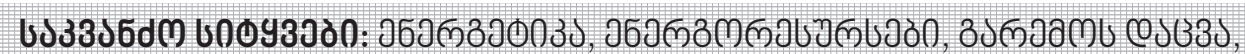

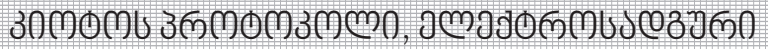

bu8mzucomgòn nб

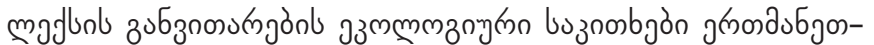

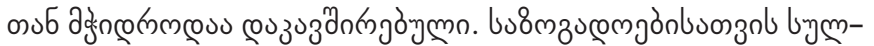
g

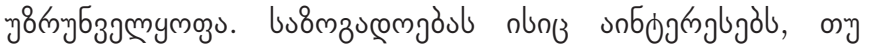

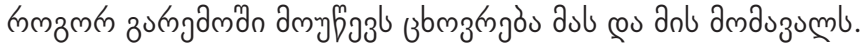

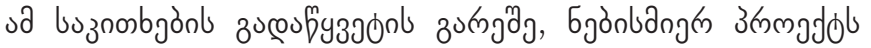

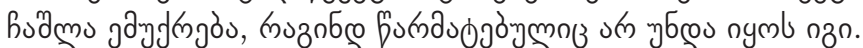

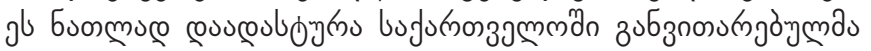

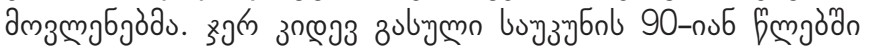

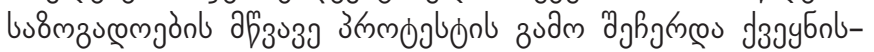

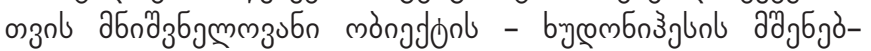

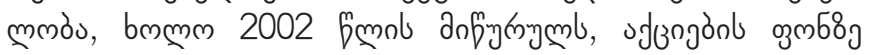

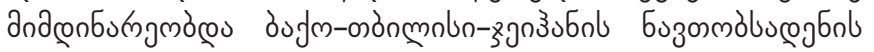

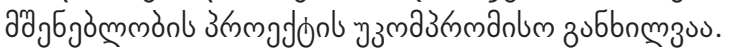

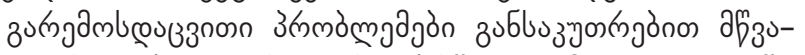

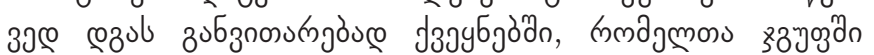

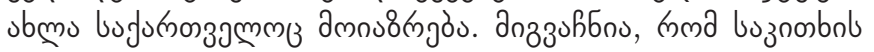

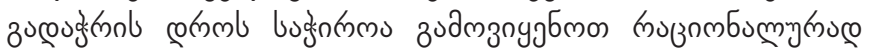

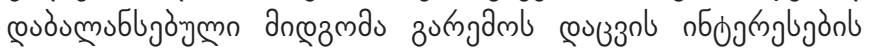

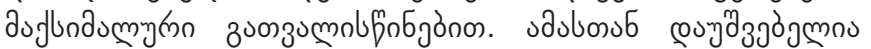

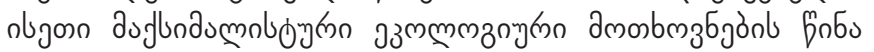

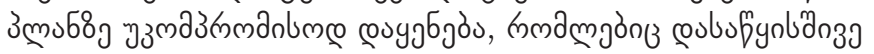

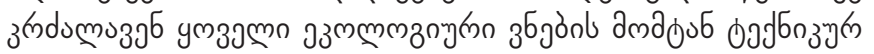
उmmgdol.

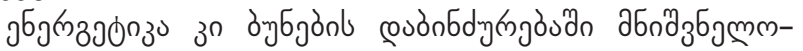

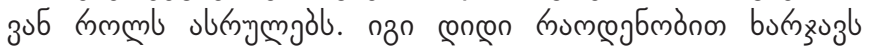

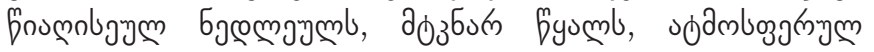

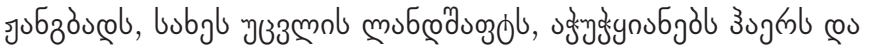

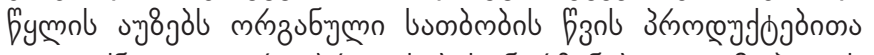

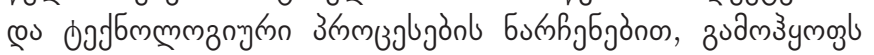

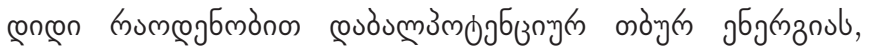

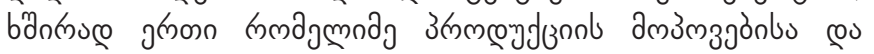

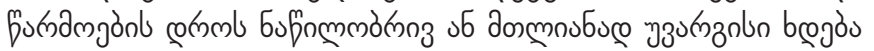

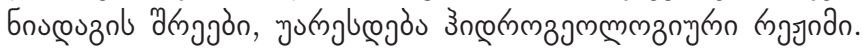

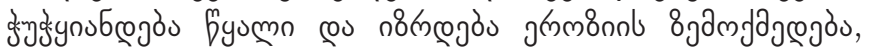

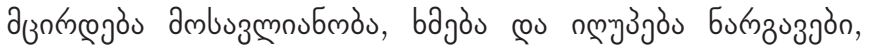

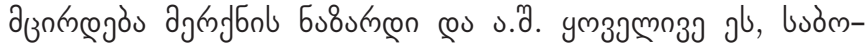

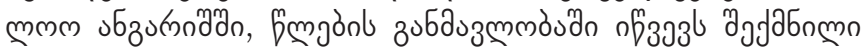

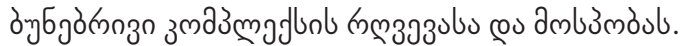

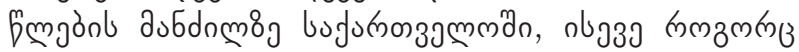

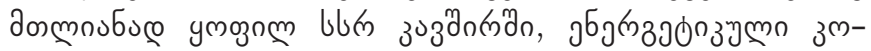

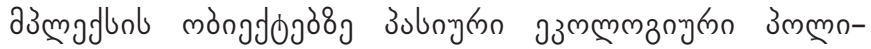

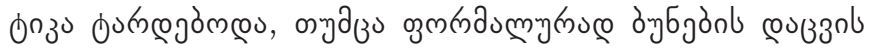

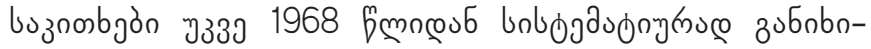

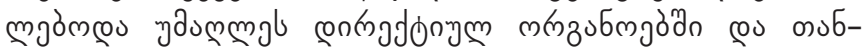

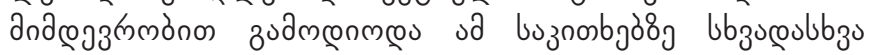

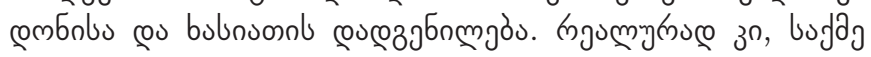

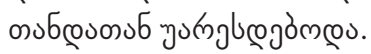

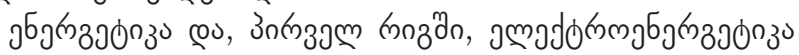
fymnl aбпдзб

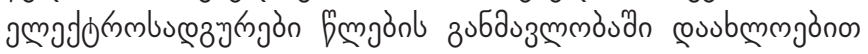

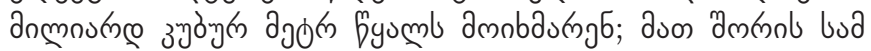

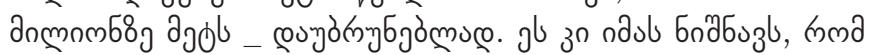

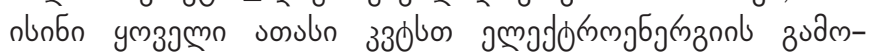

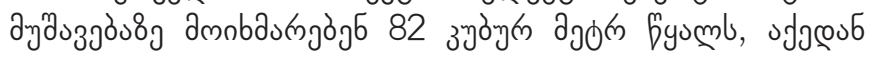

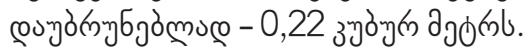

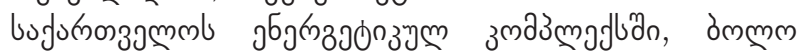

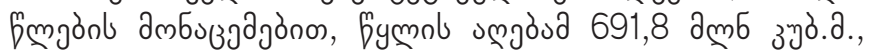

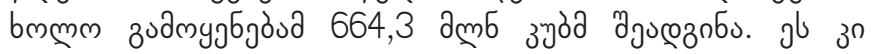

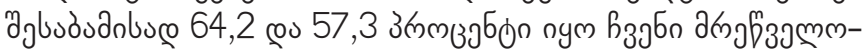

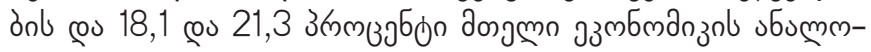

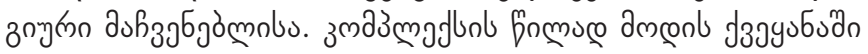

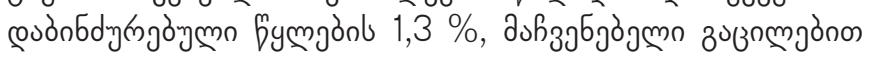
ajono a n

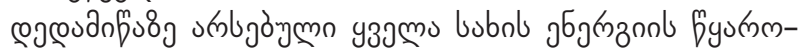

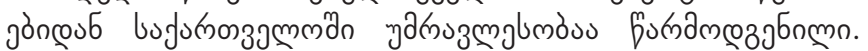

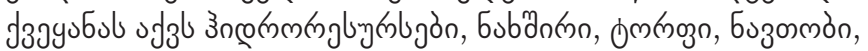
зचб

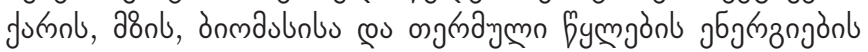
zudmygbjönbuonzol.

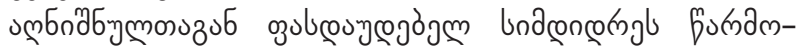

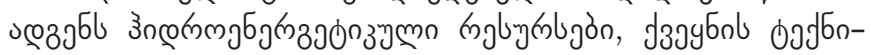

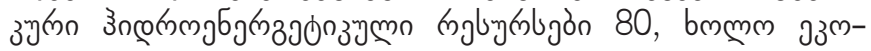




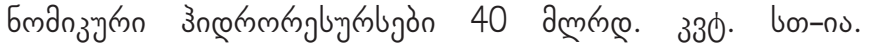

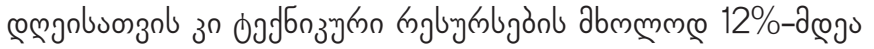

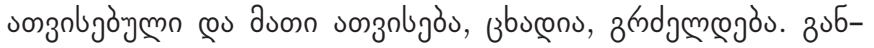

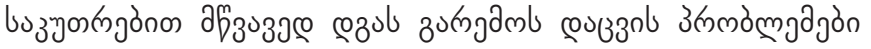

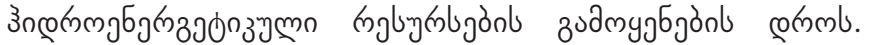

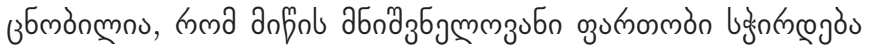

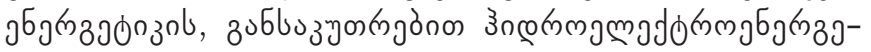

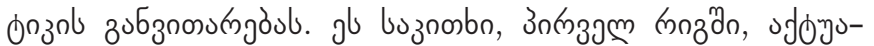

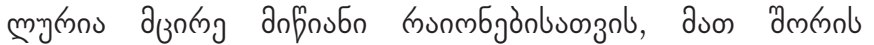

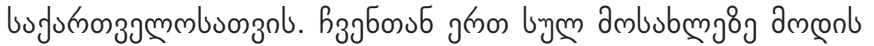

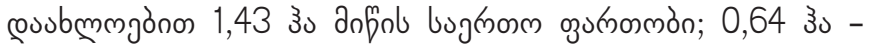

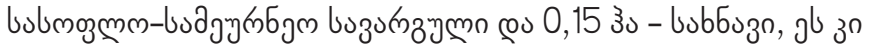
dumou 5 a zon

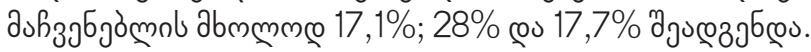

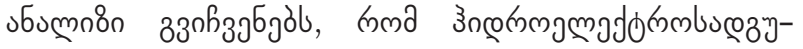

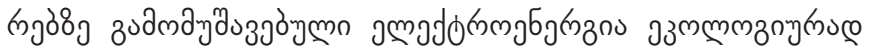

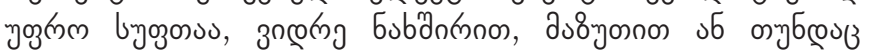

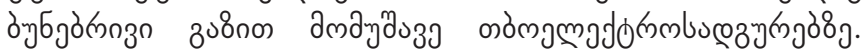

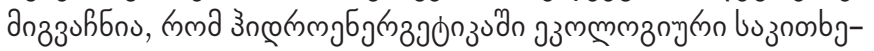

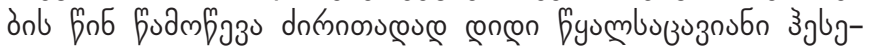

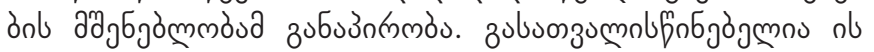

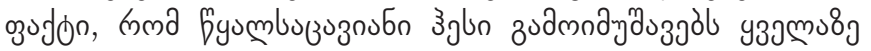

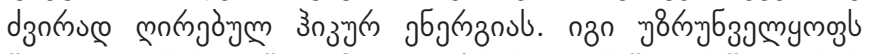

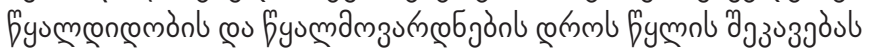

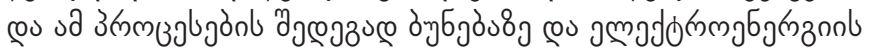

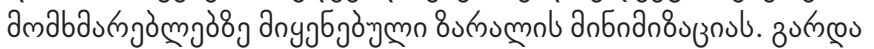

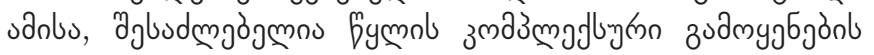

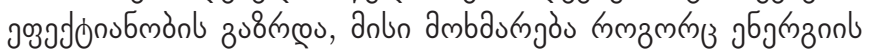

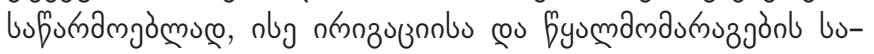

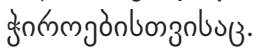

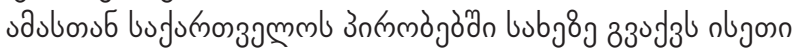

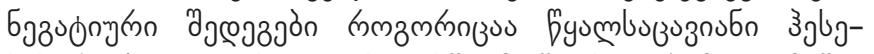

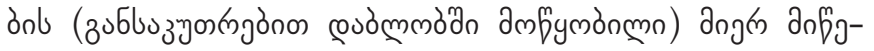

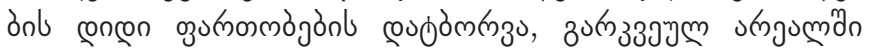

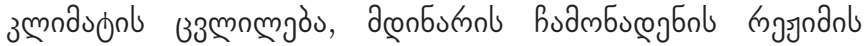

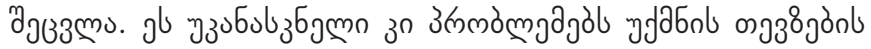

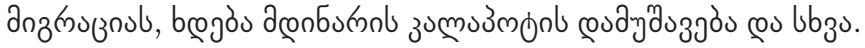

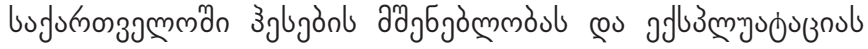

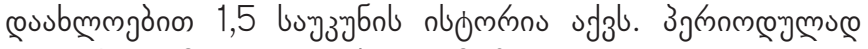

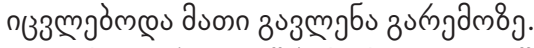

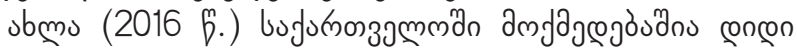

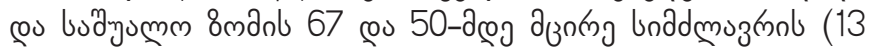

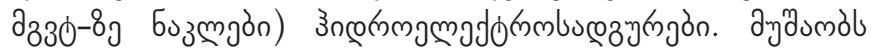

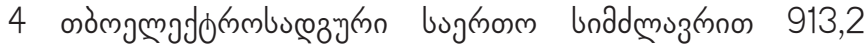

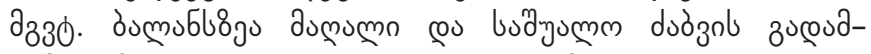

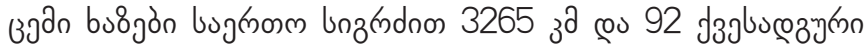

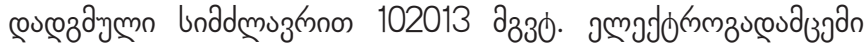

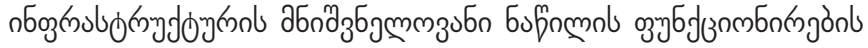

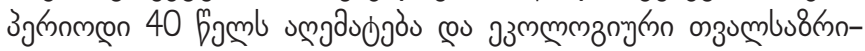

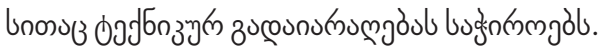

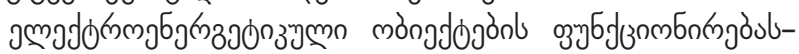

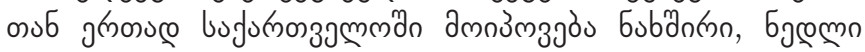

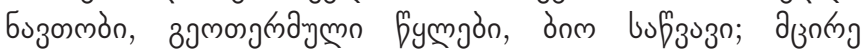

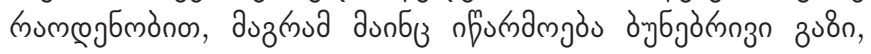
abob, jumol gб grman.

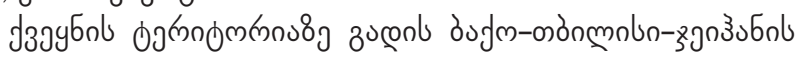

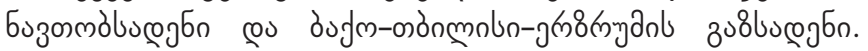

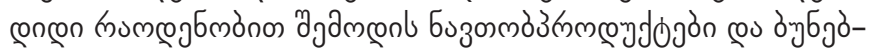

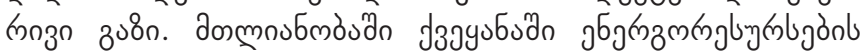

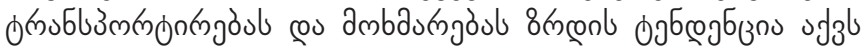

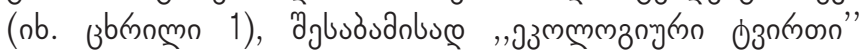

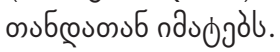

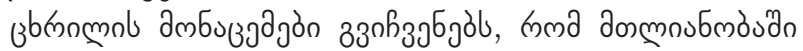

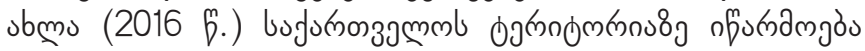

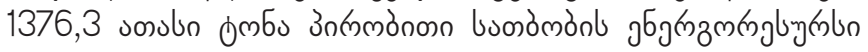

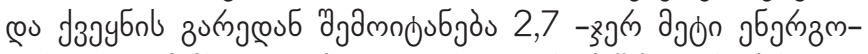

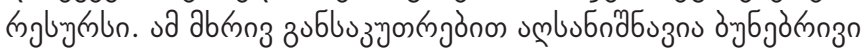

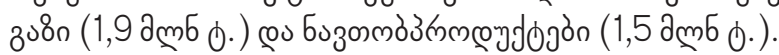

उbmomo 1.

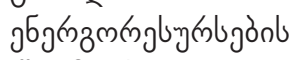

fundmgos gos

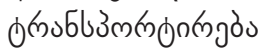

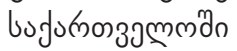

(sosulon ombs

Euzomónls

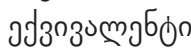

\begin{tabular}{|c|c|c|c|c|c|}
\hline 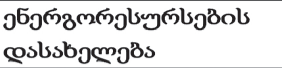 & 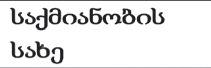 & 20146. & 20156 & 2016 f. & $\begin{array}{l}2016 \text { f. \%- } \\
2014 \text { f-oss6 }\end{array}$ \\
\hline \multirow[t]{2}{*}{ Бsbə̃omo } & fзмдмgдs & 121,5 & 124,2 & 120,4 & 99,1 \\
\hline & 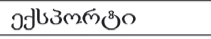 & 1,0 & 0,7 & 0,6 & 60,0 \\
\hline \multirow{3}{*}{ Бэсомп бззомїо } & fsпдmgas & 43,3 & 40,8 & 39,1 & 90,3 \\
\hline & 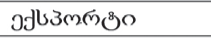 & 52,0 & 155,0 & 18,4 & 35,4 \\
\hline & ০னЗмங் & _- & 135,3 & - & - \\
\hline \multirow{3}{*}{ 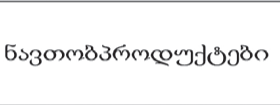 } & одЗм๓னిం & 1152,2 & 1382,5 & 1526,6 & 132,5 \\
\hline & 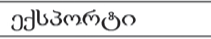 & 16,0 & 83,6 & 108,2 & 676,3 \\
\hline & 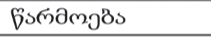 & - & 11,6 & - & - \\
\hline \multirow[t]{2}{*}{ 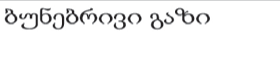 } & ంазм๓ன் & 1825,3 & 2090,6 & 1885,3 & 103,3 \\
\hline & бзмдамjos & 8,6 & 9,5 & 5,5 & 152,7 \\
\hline 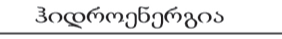 & 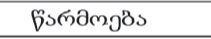 & 716,7 & 726,9 & 802,2 & 111,9 \\
\hline \multirow[t]{3}{*}{ 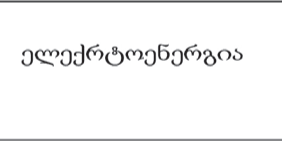 } & 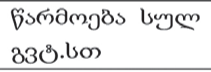 & 10371,2 & 10592,5 & 11573,6 & 111,6 \\
\hline & одзм๓ & 73,2 & 60,1 & 114,2 & 155,8 \\
\hline & 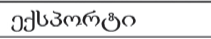 & 52,0 & 56,7 & 121,1 & 232,8 \\
\hline 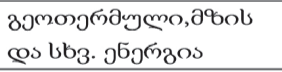 & 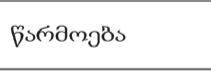 & 1,68 & 18,5 & 21,2 & 126,2 \\
\hline 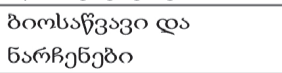 & 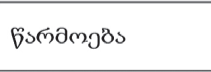 & 456,0 & 399,0 & 387,9 & 83,4 \\
\hline \multirow{3}{*}{ bynen } & 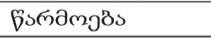 & 1372,0 & 1330,4 & 1376,3 & 100,3 \\
\hline & ంపЗмஙృిం & 3229,4 & 3820,7 & 3735,1 & 115,6 \\
\hline & 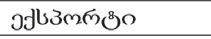 & 121,1 & 408,1 & 249,6 & 206,1 \\
\hline
\end{tabular}




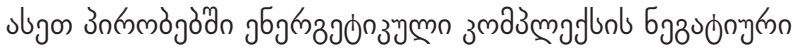

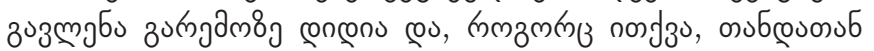

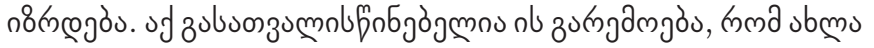

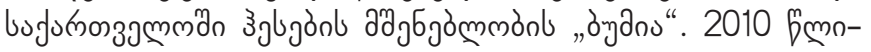

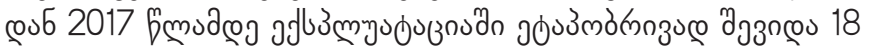

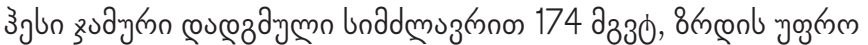

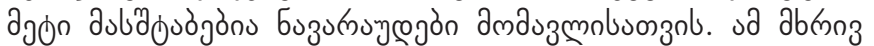

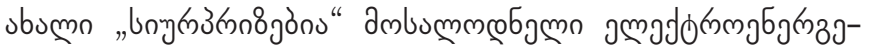

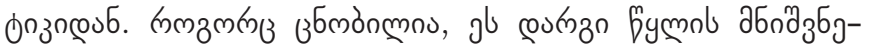

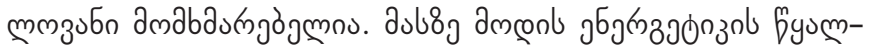

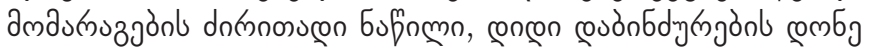

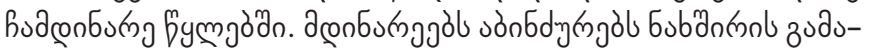

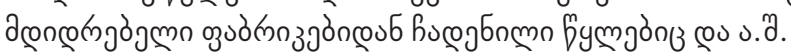

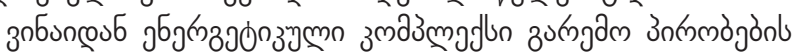
eudonбdy

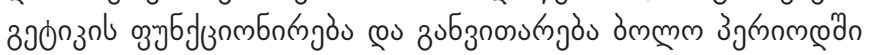

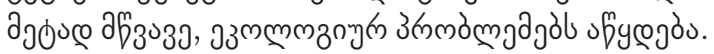

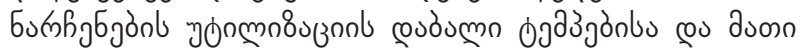

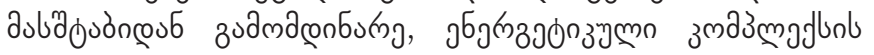

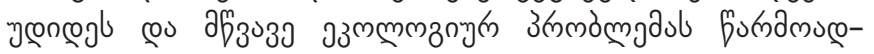

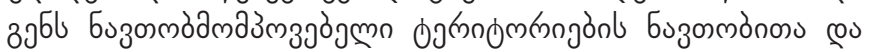

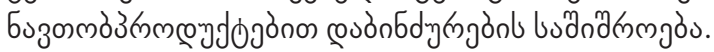

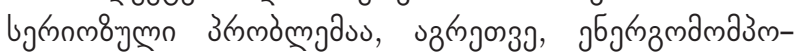

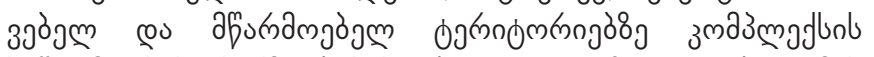

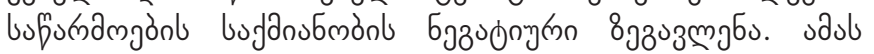

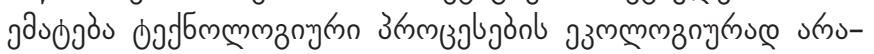

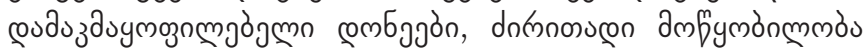

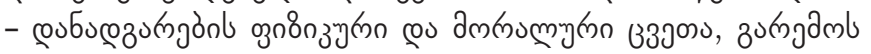

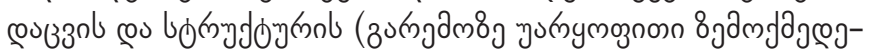

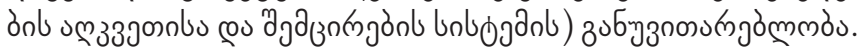

उ ऊmòm

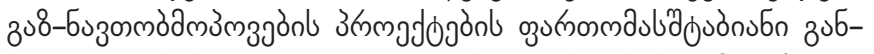

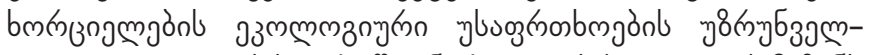

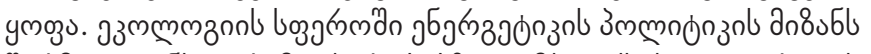

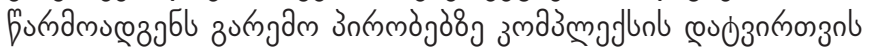

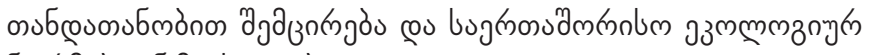

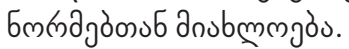

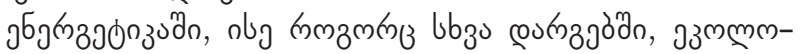

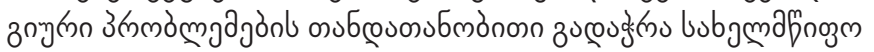

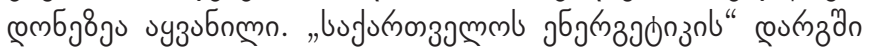

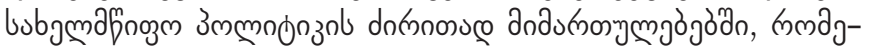

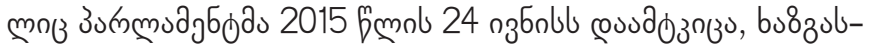
anossu zuonzum mobfoб

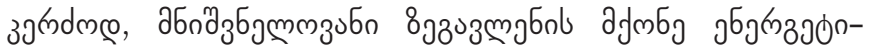

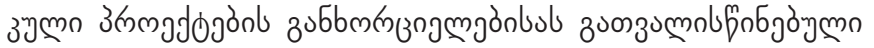
चб

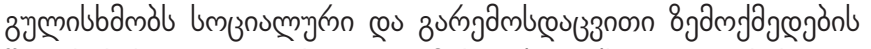

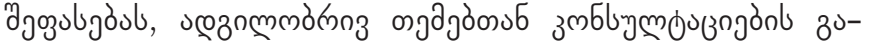

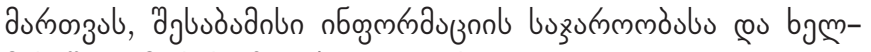

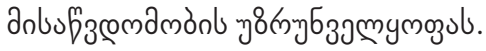

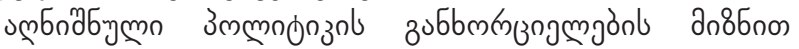

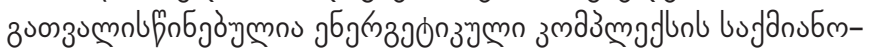

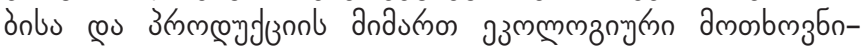

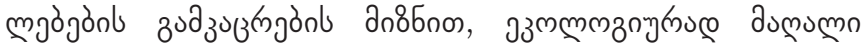

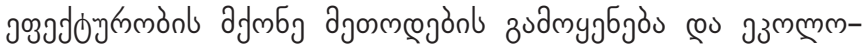

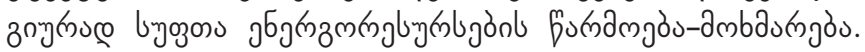

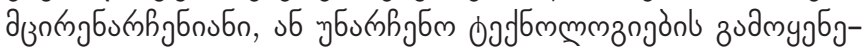

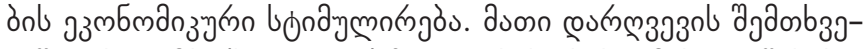

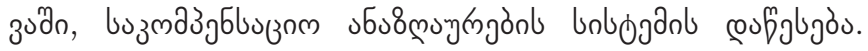

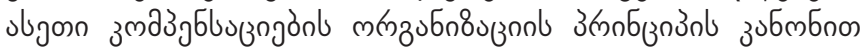

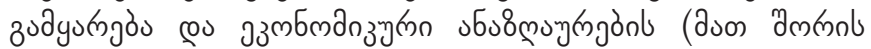

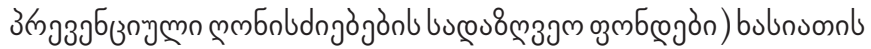

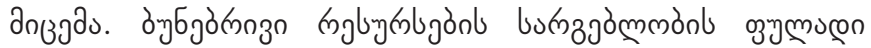

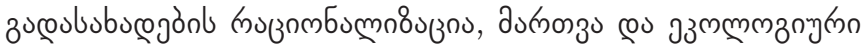

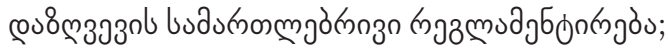

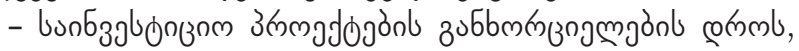

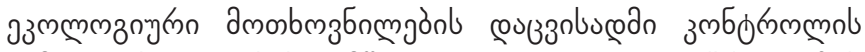

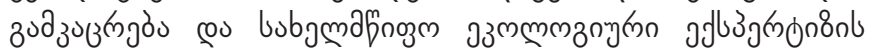
unbogan bringmymozo.

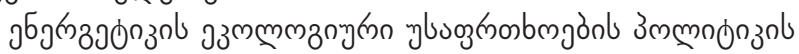

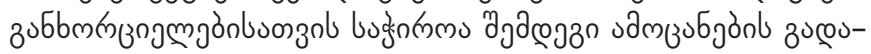
fys300:

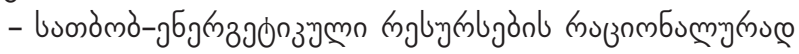

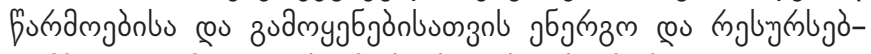

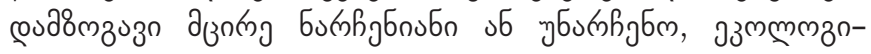

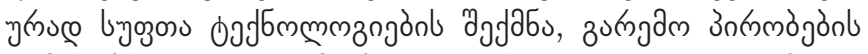

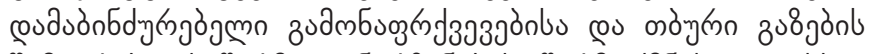

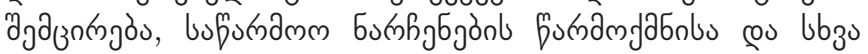

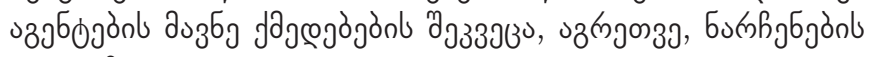
गonmoвozzo;

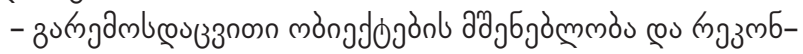

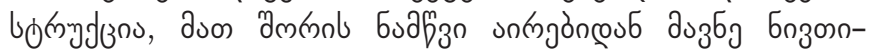

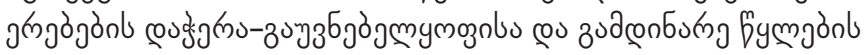

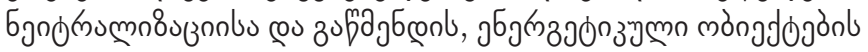

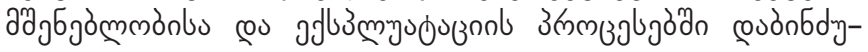

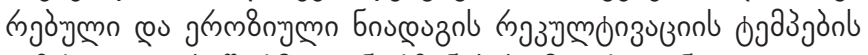

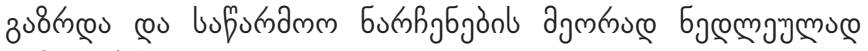
зодmgjбjos;

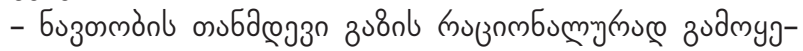

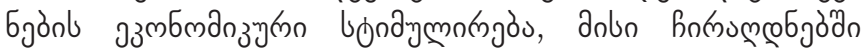

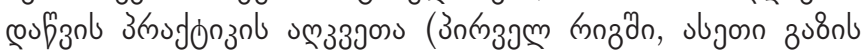

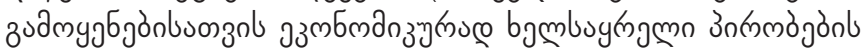

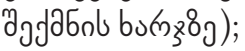

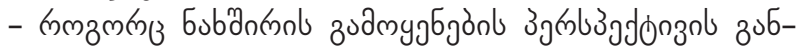

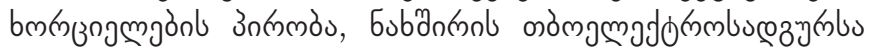

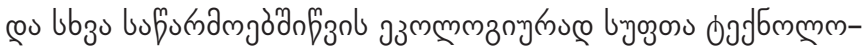

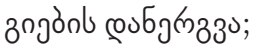

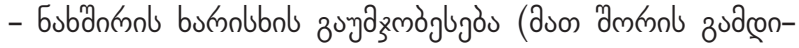

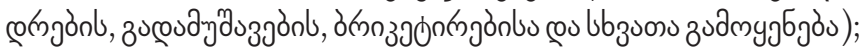

- aubojonl ajonstol zuamyjбgos;

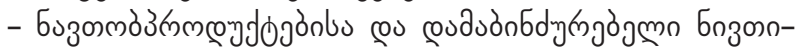

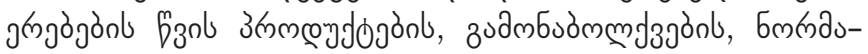

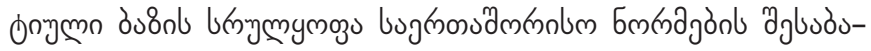

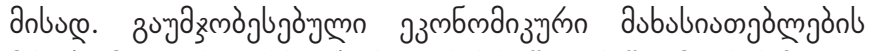

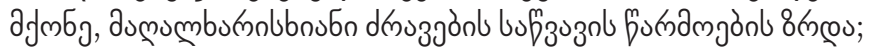

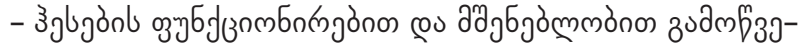




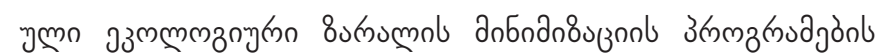

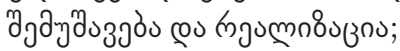

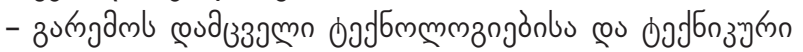

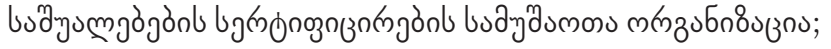

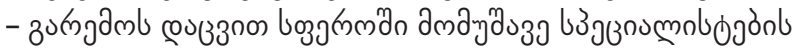

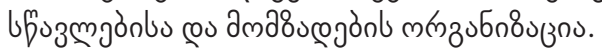

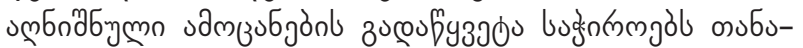

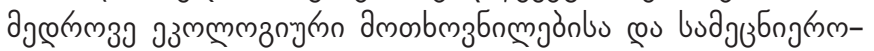

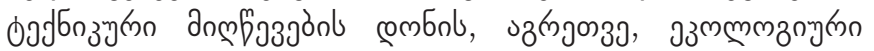

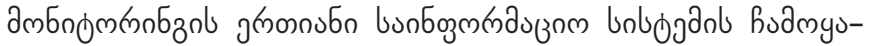

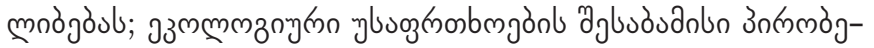

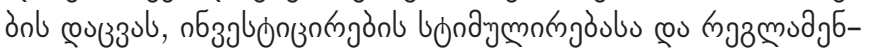

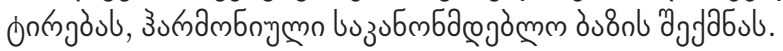

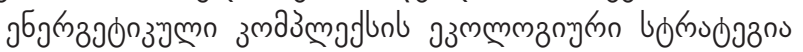

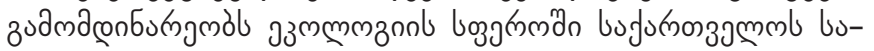

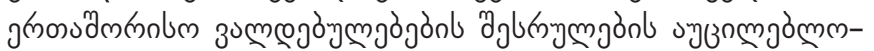

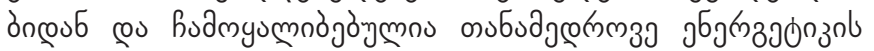

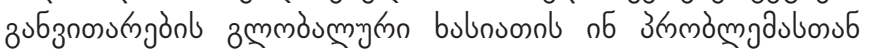

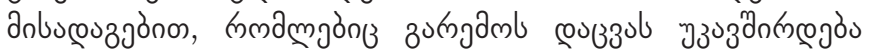

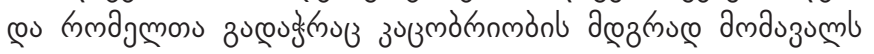

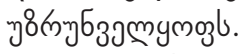

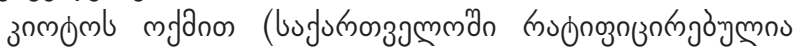

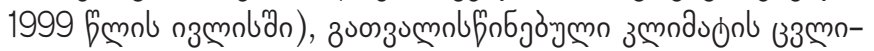

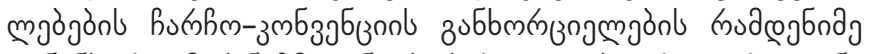

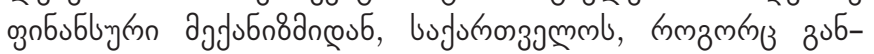

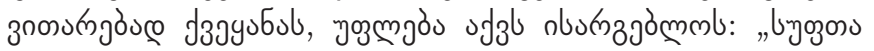

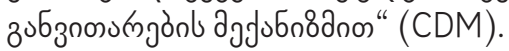

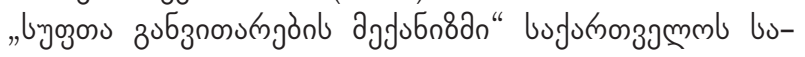

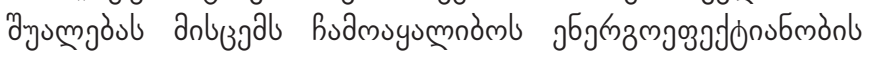

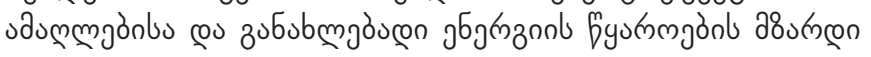

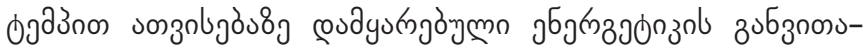

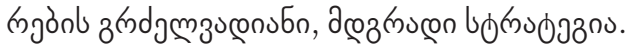

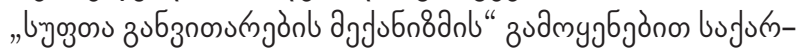

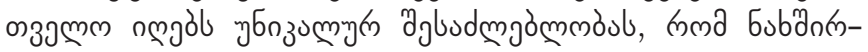

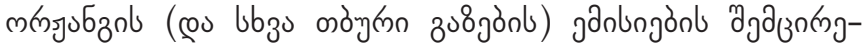

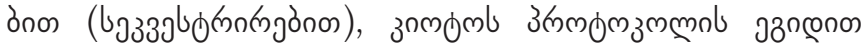

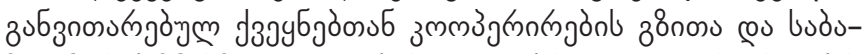

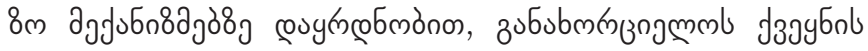

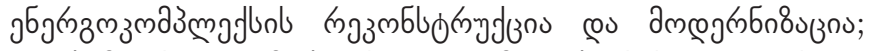

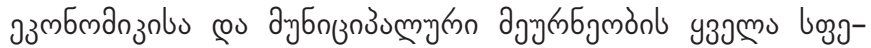

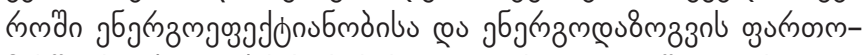

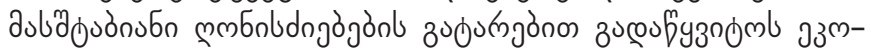

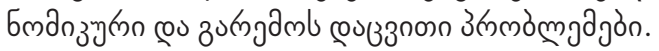

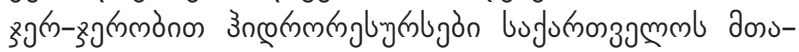
зumn jб

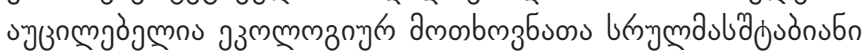

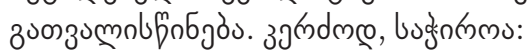

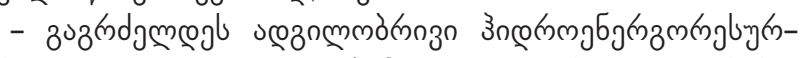

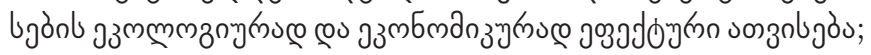

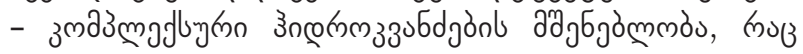

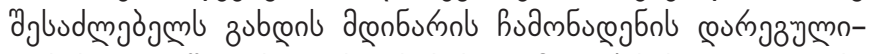

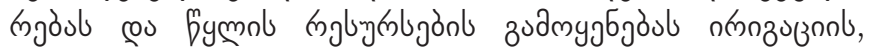

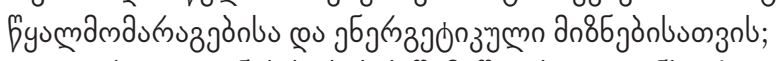

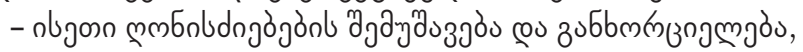

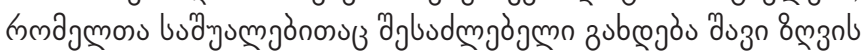

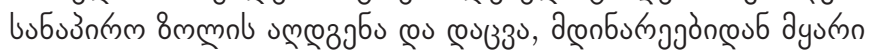

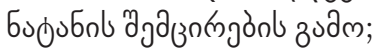

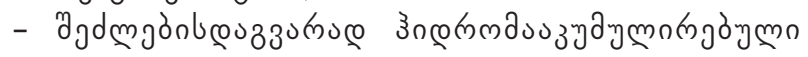

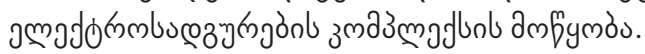

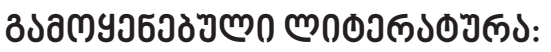

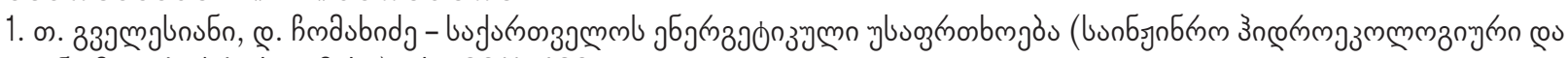

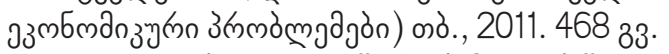

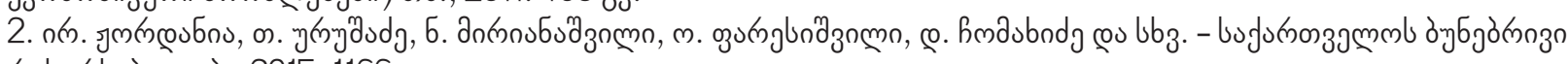

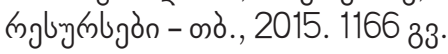

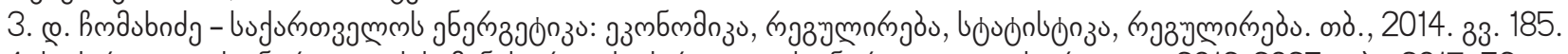

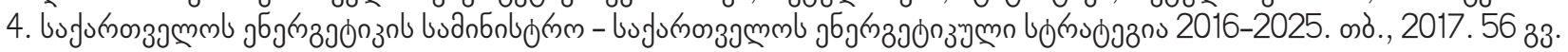

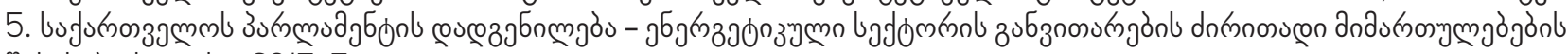
gabubjo. Jyounbon, 2015. 5 33.

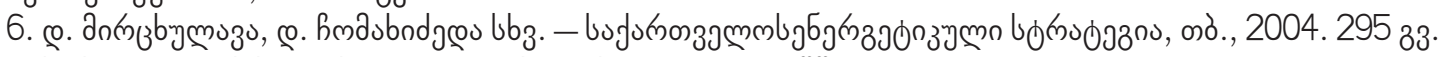

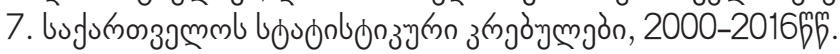

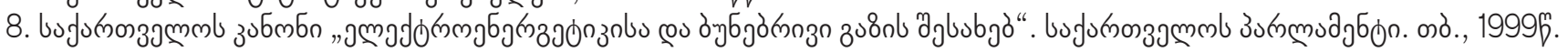

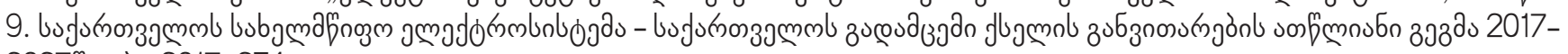

2027f. . оo., 2017. 254 33.

10. bgagzo b

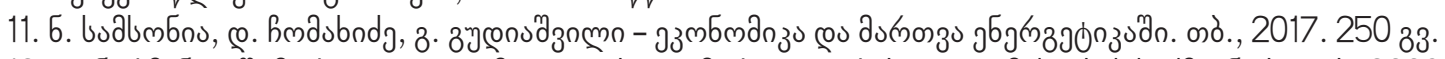

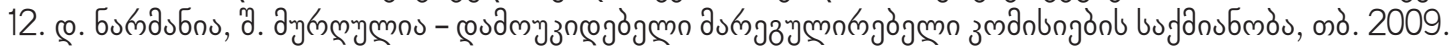

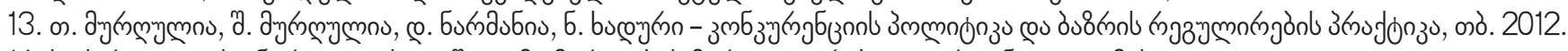

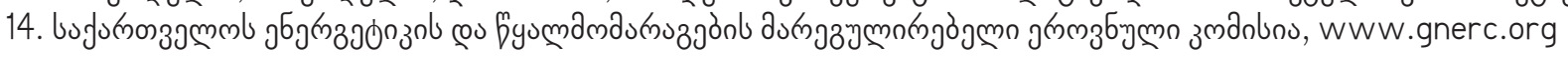

15. ChomakhidzeD. - Energy balance of Georgia, ScienceDirect, 2017.

16. ChomakhidzeD., Georgia Energy Resources; Central Asian and the Caucasus 4 (46), 2007.

17. ChomakhidzeD.,Narmania D. - Synthetic Management of Energy and Ecology in Georgia, International Journal of

Investment Management and Financial Innovations, 2018. 


\section{ECOLOGICAL CHALLENGES IN THE DEVELOPMENT OF GEORGIA ENERGY}

\section{DEMUR CHOMAKHIDZE}

https://doi.org/10.35945/gb.2018.05.001

Academic Doctor in Economics, Professor,

Georgian Technical University,

Academician of the Academy of Economic Sciences of Georgia, Georgia

d.chomakhidze@gnerc.org

\section{DAVID NARMANIA}

Academic Doctor in Economics, Professor, Ivane Javakhishvili Tbilisi State University, Georgia

davit.narmania@tsu.ge

\section{KEYWORDS: ENERGY, ENERGY RESOURCES, ENVIRONMENTAL PROTECTION, KYOTO PROTOCOL, ELECTRIC POWER STATION}

\section{SUMMARY}

The article views environmental protection issues in the energy sector of Georgia. Trends of developing energy complex are discussed and assessed envisaging ecological requirements. It is indicated that energy complex is the most polluting one among the fields of material production. Focus in made on ecological problems of the land, including cultivable ones, water, climate and other fields. The Georgian experience is presented in respect with meeting these challenges. At the end, views are given for improving the situation. Points to be stressed are:

- Rational production and usage for energy resources, creation of energy recourse-conserving, low waste or no waste environmentally clean technologies, reduction of emissions, and thermal gases polluting an environment, decrease of harmful action of industrial and other waste and waste utilization;

- Construction and reconstruction of systems of protection of environment, including increase of rates of filtration and neutralization of natural substances in the exhaust gases, waste water, cleaning of ground polluted and damaged during construction and operation of energy facilities, and also usage of industrial waste as secondary raw material;
- Provision of economic incentives of rational use of casing-head gases, suppression of practice of their burning in their torches (first of all at the experience of creation of economically favorable conditions for use of such gases);

- Introduction of environmentally clean technologies of coal burning in thermal power stations and other enterprises, as a condition of coal use prospects;

- Improvement of coal fuel quality (including its enrichment, processing, bracketing etc.);

- Usage of mine methane;

- Growth of production of high-quality engine fuels appropriate to the European standards, improved for conformity with the requirements of the advanced legal base for polluting substances and exhaust gases emitted as a result of oil products combustion;

- Development and realization of the programs of minimization of environmental damage caused by construction and functioning of hydroelectric power stations;

- Organization of works of certification of environment protection technologies and means;

- Organization of experts training working in a sphere of an environmental protection. 